Contribution from the Institut für Physikalische und Theoretische Chemie and Institut für Anorganische Chemie, Universität Regensburg, D-8400 Regensburg, FRG, and Department of Chemistry and Biochemistry, University of California, Los Angeles, California 90024

\title{
Absorption and Emission Spectra of a Single-Crystal (Maleonitriledithiolato)Palladate(II) Complex
}

\author{
Wolfgang Güntner, ${ }^{\dagger}$ Günter Gliemann, ${ }^{,+}{ }^{\dagger}$ Horst Kunkely, ${ }^{\ddagger}$ Christian Reber, ${ }^{\S}$ and Jeffrey I. Zink ${ }^{*, \S}$ \\ Received May 22, 1990 \\ The polarized optical absorption and emission of single crystals of $\left[\left(\mathrm{C}_{2} \mathrm{H}_{5}\right)_{4} \mathrm{~N}\right]_{2}\left[\mathrm{Pd}(\mathrm{mnt})_{2}\right]\left(\mathrm{mnt} \boldsymbol{\theta}\left[\mathrm{S}_{2} \mathrm{C}_{2}(\mathrm{CN})_{2}\right]^{2-}\right)$ at temperatures \\ $3 \mathrm{~K} \leq T \leq 295 \mathrm{~K}$ are reported. The lowest excited states are due to ${ }^{1,3} \mathrm{~B}_{3 \mathrm{~g}}$ and ${ }^{1,3} \mathrm{~B}_{2 u}$ terms with metal-to-ligand charge-transfer \\ and ligand-centered character, respectively. Vibronic structure is observed on the lowest energy absorption band and on the emission \\ band. The energy spacings and the intensities of the vibronic bands are analyzed.
}

\section{Introduction}

Recently polarized electronic spectra of single-crystal maleonitriledithiolate complexes $\left[\mathrm{M}(\mathrm{mnt})_{2}\right]^{2-}$ with $\mathrm{M}=\mathrm{Ni}^{1,2}$ and $\mathrm{Pt}^{3,4}$ at low temperatures have been reported. For the corresponding Pd(II) complex (cf. Figure 1) only spectroscopic data of the complex dissolved in acetonitrile ${ }^{5}$ and the complex in $\mathrm{KBr}$ disks $^{6}$ are known. The aim of this paper is to classify the optical properties and the energy levels of the palladium compound within the system Ni, Pd, Pt. For that purpose the polarized optical absorption and emission spectra of single-crystal $\left[\left(\mathrm{C}_{2} \mathrm{H}_{5}\right)_{4} \mathrm{~N}\right]_{2^{-}}$. $\left[\operatorname{Pd}(\mathrm{mnt})_{2}\right]$ has been measured under variation of temperature. By comparison of the results with those of the $\mathrm{Ni}$ and $\mathrm{Pt}$ complexes, an energy level diagram of the lowest electronic states of $\left[\operatorname{Pd}(\mathrm{mnt})_{2}\right]^{2-}$ is proposed.

Vibronic structure in the emission spectrum is observed and analyzed. Detailed fitting of the emission spectrum yields the values of the distortions along 11 normal coordinates. The analysis supports the orbital assignments of the lowest excited state.

\section{Experimental Section}

The compound $\left[\left(\mathrm{C}_{2} \mathrm{H}_{5}\right)_{4} \mathrm{~N}\right]_{2}\left[\mathrm{Pd}(\mathrm{mnt})_{2}\right]$ was prepared according to a known method.' Single crystals were obtained by slow evaporation of acetonitrile solution at $\sim 5^{\circ} \mathrm{C}$

The absorption and emission measurements were performed with apparatus described in refs $8-10$. The emission spectra are corrected for the spectral response of the detecting system.

\section{Results}

Single-crystal $\left[\left(\mathrm{C}_{2} \mathrm{H}_{5}\right)_{4} \mathrm{~N}\right]_{2}\left[\mathrm{Pd}(\mathrm{mnt})_{2}\right]$ exhibits a distinct dichroism. The dichroic axis $\mathbf{A}$ has been used to define the orientation of the single crystal relative to the polarization geometry of the apparatus.

The absorption spectrum of $\left[\left(\mathrm{C}_{2} \mathrm{H}_{5}\right)_{4} \mathrm{~N}\right]_{2}\left[\mathrm{Pd}(\mathrm{mnt})_{2}\right]$ at room temperature is $\mathbf{E} \| \mathbf{A}$ polarized and shows a strong band with maximum at $\bar{\nu}=21700 \mathrm{~cm}^{-1}$ and a shoulder at its high energy flank; cf. Figure 2 (top). A corresponding band with $\epsilon \sim 5700$ $\mathrm{M}^{-1} \mathrm{~cm}^{-1}$ has been observed for $\left[\left(\mathrm{C}_{2} \mathrm{H}_{5}\right)_{4} \mathrm{~N}\right]_{2}\left[\mathrm{Pd}(\mathrm{mnt})_{2}\right]$ dissolved in acetonitrile. ${ }^{5}$ Upon decrease of the temperature to $T=10 \mathrm{~K}$, the strong absorption band splits into several peaks and shoulders (cf. Figure 2 (bottom)), whose spectral positions are summarized in Table I. The integral extinction of the band, however, shows no dependence on the temperature.

At $\bar{\nu} \sim 15700 \mathrm{~cm}^{-1}$, an additional very weak $\mathbf{E} \| \mathbf{A}$ polarized absorption of single-crystal $\left[\left(\mathrm{C}_{2} \mathrm{H}_{5}\right)_{4} \mathrm{~N}\right]_{2}\left[\mathrm{Pd}(\mathrm{mnt})_{2}\right]$ has been detected. (An acetonitrile solution of $\left[\left(\mathrm{C}_{2} \mathrm{H}_{5}\right)_{4} \mathrm{~N}\right]_{2}\left[\mathrm{Pd}(\mathrm{mnt})_{2}\right]$ also shows an absorption with $\epsilon \sim 64 \mathrm{M}^{-1} \mathrm{~cm}^{-1}$ as this wavelength..$^{5}$ ) Between room temperature and $T=10 \mathrm{~K}$, the intensity of the $15700-\mathrm{cm}^{-1}$ band decreases by a factor of about 8 and the band maximum is blue-shifted by $\Delta \bar{\nu} \sim 200 \mathrm{~cm}^{-1}$. At low temperatures, fine structure at the red flank of the band can be resolved; cf. Figure 3. The lines forming the fine structure have half-widths of about $7 \mathrm{~cm}^{-1}$. The very weak origin at $\bar{\nu}=14670 \mathrm{~cm}^{-1}$ is

\footnotetext{
'Institut für Physikalische und Theoretische Chemie, Universität Regensburg.

Institut für Anorganische Chemie, Universität Regensburg.

IUniversity of California.
}

Table I. Absorption Bands of Single-Crystal $\left[\left(\mathrm{C}_{2} \mathrm{H}_{5}\right)_{4} \mathrm{~N}\right]_{2}\left[\mathrm{Pd}(\mathrm{mnt})_{2}\right]$ at $T=10 \mathrm{~K}^{a}$

\begin{tabular}{lccl}
\hline $\begin{array}{c}\text { energetic } \\
\text { posn, } \mathrm{cm}^{-1}\end{array}$ & $\begin{array}{c}\text { energy sepn from } \\
\text { the } 21180-\mathrm{cm}^{-1} \\
\text { band, } \mathrm{cm}^{-1}\end{array}$ & $\begin{array}{c}\text { Raman } \\
\text { band, } \mathrm{cm}^{-1}\end{array}$ & \multicolumn{1}{c}{ assgnt } \\
\hline 21180 & & & ${ }^{1} \mathrm{~A}_{\mathrm{g}} \rightarrow{ }^{1} \mathrm{~B}_{2 \mathrm{u}}$ \\
21660 & 480 & 494.6 & $+\nu$ \\
22140 & 960 & & $+2 \nu(494.6)$ \\
22580 & 1400 & 1482 & $+\nu(\mathrm{C}=\mathrm{C})$ \\
23300 & 2120 & 2195 & $+\nu(\mathrm{C}=\mathrm{N})$ \\
$23780 \mathrm{sh}$ & 2600 & & $+\nu(\mathrm{C}=\mathrm{N})+(494.6)$ \\
24700 & 3520 & & $+\nu(\mathrm{C}=\mathrm{N})+\nu(\mathrm{C}=\mathrm{C})$ \\
$25420 \mathrm{sh}$ & 4240 & & $+2 \nu(\mathrm{C} \equiv \mathrm{N})$
\end{tabular}

${ }^{\circ}$ All Raman bands belong to totally symmetric molecular vibrations. sh $=$ shoulder.

followed by a sharp and relatively intense peak $16 \mathrm{~cm}^{-1}$ at higher energy. The spectrum exhibits a dominating $150-\mathrm{cm}^{-1}$ progression superimposed by an additional progression with $\Delta \bar{\nu}=16 \mathrm{~cm}^{-1}$. Between the intense line of highest energy in each group of lines and the adjacent peak of the neighboring series of low-intensity lines, there is an energy gap of $\Delta \bar{\nu}=28 \mathrm{~cm}^{-1}$.

The emission spectrum is partly polarized with an intensity ratio $I_{\perp} / I_{\|}$of about 2 , independent of the wavelength. At $T \sim 3 \mathrm{~K}$, the emission spectrum (cf. Figure 4) shows fine structure similar to the one found in the low-energy absorption; the two progressions, however, have slightly higher energies, $\Delta \bar{\nu}=26$ and $162 \mathrm{~cm}^{-1}$, respectively. A conspicuous result is the large energy gap $\Delta \vec{\nu}=$ $2190 \mathrm{~cm}^{-1}$ between the absorption peak of lowest energy $(\bar{\nu}=$ $\left.14670 \mathrm{~cm}^{-1}\right)$ and the emission peak of highest energy $(\bar{\nu}=12480$ $\mathrm{cm}^{-1}$ ). With increasing temperature, the emission acquires intensity; the fine structure of the emission spectrum, however, disappears.

\section{Discussion}

Assignment of the Excited Electronic States. The crystal structure of $\left[\left(\mathrm{C}_{2} \mathrm{H}_{5}\right)_{4} \mathrm{~N}\right]_{2}\left[\mathrm{Pd}(\mathrm{mnt})_{2}\right]$ is not known as yet. On the basis of structure data for $\mathrm{K}\left[\mathrm{Pd}(\mathrm{mnt})_{2}\right] \cdot \mathrm{H}_{2} \mathrm{O}$ and $\left(\mathrm{NH}_{4}\right)[\mathrm{Pd}-$ (mnt) $\left.{ }_{2}\right] \cdot \mathrm{H}_{2} \mathrm{O}$, which are isostructural with the corresponding $\mathrm{Ni}$ and $\mathrm{Pt}$ complexes, ${ }^{11}$ and for $\left[\left(\mathrm{C}_{4} \mathrm{H}_{9}\right)_{4} \mathrm{~N}\right]_{2}\left[\mathrm{M}(\mathrm{mnt})_{2}\right]$ with $\mathrm{M}=$ $\mathrm{Ni}^{12}$ and $\mathrm{M}=\mathrm{Pt}^{3}{ }^{3}$ it can be assumed that the anion $\left[\mathrm{Pd}(\mathrm{mnt})_{2}\right]^{2-}$

(1) Chandramouli, G. V. R.; Manoharan, P. T. Inorg. Chem. 1986, 25 , 4680.

(2) Lalitha, S.; Chandramouli, G. V. R.; Manoharan, P. T. Inorg. Chem. 1988, 27, 1492.

(3) Güntner, W.; Gliemann, G.; Klement, U.; Zabel, M. Inorg. Chim. Acta $1989,165,51$

(4) Güntner, W.; Gliemann, G. J. Phys. Chem. 1990, 94, 618.

(5) Shupack, S. I.; Billig, E.; Clark, R. J. H.; Williams, R.; Gray, H. B. J. Am. Chem. Soc. 1964, 86, 4594 .

(6) Clark, R. J. H.; Turtle, P. C. J. Chem. Soc., Dalton Trans. 1977, 2142.

(7) Bähr, G.; Schleitzer, G. Chem. Ber. 1957, 90, 438.

(8) Tuszynski, W.; Gliemann, G. Ber. Bunsen-Ges. Phys. Chem. 1985, 89. 940.

(9) Yersin, H.; Gliemann, G. Messtechnik 1972, 80, 99.

(10) Gliemann, G. Comments Inorg. Chem. 1986, 5, 263.

(11) Hursthouse, M. B.; Short, R. L.; Clemenson, P. I.; Underhill, A. E. J. Chem. Soc., Dalton Trans. 1989, 67 
<smiles>CC(C)(C)S[Te]1(I)(SC(C)(C)C)SC(C#N)=C(C#N)S1</smiles>

Figure 1. Schematic structure of the $\left[\mathrm{Pd}(\mathrm{mnt})_{2}\right]^{2-}$ complex ion and the molecular axes.

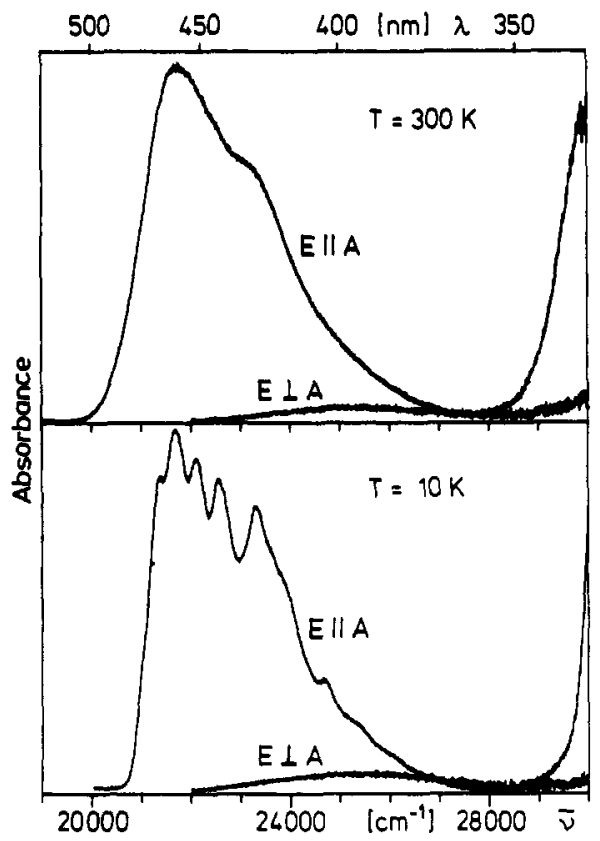

Figure 2. Polarized absorption spectra of single-crystal $\left[\left(\mathrm{C}_{2} \mathrm{H}_{5}\right)_{4} \mathrm{~N}\right]_{2}$. $\left[\mathrm{Pd}(\mathrm{mnt})_{2}\right]$ at $T=300 \mathrm{~K}$ and $T=10 \mathrm{~K}$.

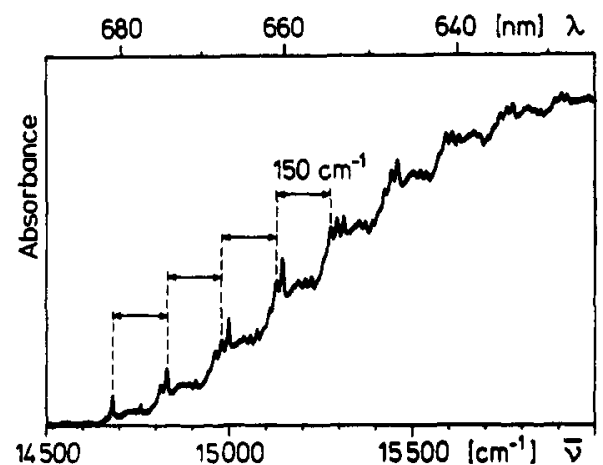

Figure 3. Low-energy section of the $\mathbf{E} \| \mathbf{A}$ polarized absorption spectrum of single-crystal $\left[\left(\mathrm{C}_{2} \mathrm{H}_{5}\right)_{4} \mathrm{~N}\right]_{2}\left[\mathrm{Pd}(\mathrm{mnt})_{2}\right]$ at $T=10 \mathrm{~K}$.

in $\left[\left(\mathrm{C}_{2} \mathrm{H}_{5}\right)_{4} \mathrm{~N}\right]_{2}\left[\mathrm{Pd}(\mathrm{mnt})_{2}\right]$ also has a nearly planar structure and the $\mathrm{Pd}$ atom is surrounded by the four $\mathrm{S}$ atoms at the corners of a rectangle. Thus, the symmetry of the $\mathrm{Pd}$ atom with its nearest neighbors can be described by the point group $D_{2 h} ;$ cf. Figure 1. In the crystal, the anions are expected to be distinctly separated by the voluminous counterions. ${ }^{3,12}$ The crystal optical properties of $\left[\left(\mathrm{C}_{2} \mathrm{H}_{5}\right)_{4} \mathrm{~N}\right]_{2}\left[\mathrm{Pd}(\mathrm{mnt})_{2}\right]$ are very similar to those of the corresponding platinum compound. ${ }^{4}$ Therefore, the orientation of the dichroic axis $\mathbf{A}$ is expected to be parallel to the molecular $y$ axis.

For the assignment of the optical transitions, the results of a Wolfsberg-Helmholz MO calculation for the $\left[\mathrm{Ni}\left(\mathrm{S}_{2} \mathrm{C}_{2} \mathrm{H}_{2}\right)_{2}\right]$ complex can be used. ${ }^{13}$ In refs 1 and 5 the following energy order

(12) Kobayashi, A.: Sasaki, Y. Bull. Chem. Soc. Jpn. 1977, 50, 2650.

(13) Schrauzer, G. N.; Mayweg, V. P. J. Am. Chem. Soc. 1965, 87, 3585.

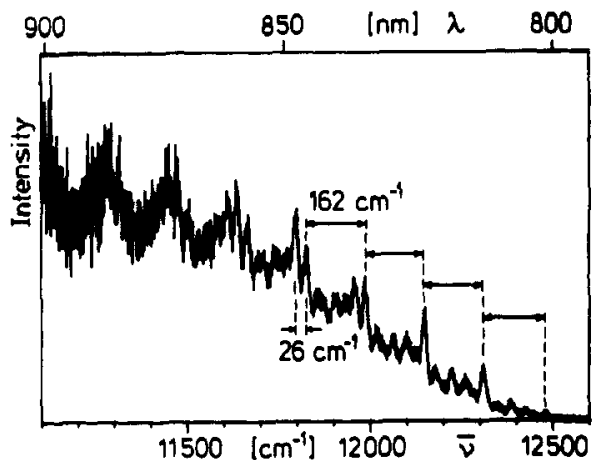

Figure 4. E $\perp$ A polarized emission spectrum of single-crystal $\left[\left(\mathrm{C}_{2} \mathrm{H}_{5}\right)_{4} \mathrm{~N}\right]_{2}\left[\mathrm{Pd}(\mathrm{mnt})_{2}\right]$ at $T \approx 3 \mathrm{~K} . \lambda_{\text {exc }}=364 \mathrm{~nm}$.

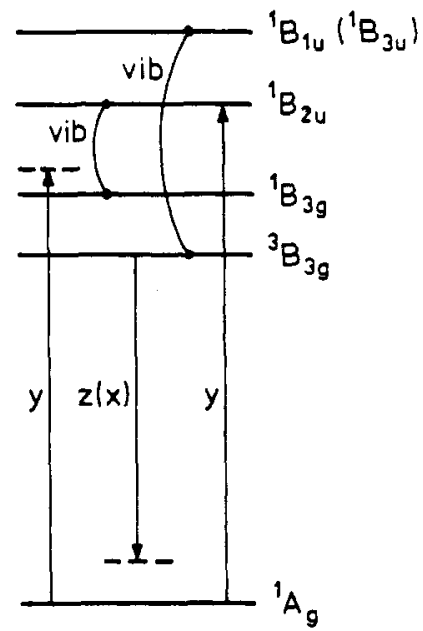

Figure 5. Proposed energy level diagram of the lowest electronic states of $\left[\left(\mathrm{C}_{2} \mathrm{H}_{5}\right)_{4} \mathrm{~N}\right]_{2}\left[\mathrm{Pd}(\mathrm{mnt})_{2}\right]$.

of the one-electron levels of this complex is reported: $b_{2 g}\left(d_{x y}\right)<$ $a_{g}\left(d_{z^{2}}\right)<b_{3 g}\left(d_{y z}\right)<b_{14}\left(L_{\pi}\right)<a_{g}\left(d_{x^{2}-y^{2}}\right)<b_{2 g}\left(L_{\pi}+d_{x z}\right)<b_{1 g}\left(d_{x y}\right)$ $<\mathrm{a}_{\mathrm{u}}, \mathrm{b}_{3 \mathrm{~g}}\left(\mathrm{~L}_{\pi^{*}}\right)$.

By use of a similar energy order for the MO's, the optical spectra of single-crystal $\left[\left(\mathrm{C}_{2} \mathrm{H}_{9}\right)_{4} \mathrm{~N}\right]_{2}\left[\mathrm{Ni}(\mathrm{mnt})_{2}\right]$ could be explained.' By ESR experiments with the oxidized and reduced forms $\left[\operatorname{Pd}(\mathrm{mnt})_{2}\right]^{-}$and $\left[\mathrm{Pd}(\mathrm{mnt})_{2}\right]^{3-}$, respectively, it has been confirmed that the described energy order can be applied also to the HOMO and LUMO of the [Pd(mnt $\left.)_{2}\right]^{2-}$ complex ion. ${ }^{14.15}$ Thus, the $b_{2 g}$ MO (with overwhelming ligand character) forms the HOMO, and the LUMO transforms as $b_{1 g}$ (with $d_{x y}$ metal character).

The strong absorption band with maximum at $\bar{\nu}=21180 \mathrm{~cm}^{-1}$ (cf. Figure 2) can be assigned to the singlet-singlet transition $b_{2 g}\left(L_{\pi}+d_{x z}\right) \rightarrow a_{u}\left(L_{\pi^{*}}\right)$, i.e. ${ }^{1} A_{g} \rightarrow{ }^{1} B_{2 u}$; cf. Figure 5 . This transition is electric dipole allowed with $\mathbf{E} \| \mathbf{y}$ polarization. The structure of this band in the low-temperature spectrum (cf. Figure 2(bottom)) can be traced back to a coupling with totally symmetric $a_{g}$ vibrations; cf. Table 1 . A comparison with the similarly structured spectra of single-crystal $\left[\mathrm{Pt}(\mathrm{mnt})_{2}\right]^{2-}$ complexes $^{3,4}$ suggests that the shoulder at $\bar{\nu}=21360 \mathrm{~cm}^{-1}$ does not represent the 0-0 transition but that this transition is expected at an energy lower by $\Delta \bar{\nu} \sim 180 \mathrm{~cm}^{-1}$. The assignment of the absorption peak at $\bar{\nu}=23300 \mathrm{~cm}^{-1}$ to an additional electronic transition (cf. ref 6) looks unlikely, since the energy separation between this peak and the absorption edge of the strong band equals nearly that of the corresponding platinum complexes. 3,4

The weak absorption at $\bar{\nu} \sim 15700 \mathrm{~cm}^{-1}$ can be assigned to the vibronic transition ${ }^{1} A_{g} \rightarrow{ }^{1} B_{3 g}$, due to the symmetry-forbidden $b_{2 \mathrm{~g}} \rightarrow b_{1 \mathrm{~g}}$ excitation. By vibronic coupling of the states ${ }^{1} B_{3 \mathrm{~g}}$ and ${ }^{\prime} B_{2 u}$, this transition acquires oscillator strength; $\mathrm{cf}$. Figure 5 . As

(14) Kirmse, R.; Dietzsch, W. J. Inorg. Nucl. Chem. 1976, 38, 255.

(15) Geiger, W. E., Jr.; Allen, C. S.; Mines, T. E.; Senftleber, F. C. Inorg. Chem. 1977, 16, 2003. 
a consequence of this assignment, the absorption peak at $\bar{\nu}=14670$ $\mathrm{cm}^{-1}$ represents a false origin.

The emission is due to the electronic transition ${ }^{3} B_{3 g} \rightarrow{ }^{1} A_{g}$, which is both spin and symmetry forbidden. (Obviously the corresponding absorption process is too weak to be detectable.) The emission acquires intensity by spin-orbit and vibronic coupling of the state ${ }^{3} B_{3 g}$ with excited singlets. Since the emission is only partly polarized, other singlets in addition to singlets of $B_{2 u}$ symmetry will be involved. The vibronic character of the emission and of the low-energy absorption manifests itself by (i) the large energy gap $\left(\Delta \bar{\nu} \sim 2190 \mathrm{~cm}^{-1}\right)$ between the absorption and emission and (ii) the enhancement of the extinction (absorption) and the intensity (emission) with increasing temperature.

The Time-Dependent Theory of Emission Spectroscopy. The time-dependent theory of electronic spectroscopy provides a quantitative method of calculating the intensities of the vibronic structure in emission and absorption spectra. In addition, it provides a simple explanation for the repetitive pattern of small spacings in the emission spectrum. Because the theory has been discussed in detail previously, ${ }^{16-19}$ only a brief discussion of the general aspects will be given here.

The spectra are governed by the motion of a wavepacket on the multidimensional electronic state potential surface. The initial wavepacket, $\phi$, is projected onto the ground-state potential energy surface. This surface is, in general, displaced relative to the initial surface, and the wavepacket begins to move, following the path of steepest descent. The wavepacket is not a stationary state of the final surface and evolves according to the time-dependent Schrödinger equation. The emission spectrum is given by ${ }^{6}$

$$
I(\omega)=C \omega^{3} \int_{-\infty}^{\infty} e^{j \omega t}\langle\phi \mid \phi(t)\rangle \mathrm{d} t
$$

where $C$ is a constant and $I(\omega)$ is the intensity in photons per unit volume per unit time at frequency of emitted radiation $\omega$. The quantity $\langle\phi \mid \phi(t)\rangle$ is the overlap of the initial wavepacket, $\phi(0)$, with the time-dependent wavepacket, $\phi(t)$. The absorption spectrum is calculated by using a similar expression except that $\omega^{3}$ is replaced by $\omega$.

If it is assumed that (a) the normal coordinates are not mixed in the excited state, (b) the transition dipole moment, $\mu$, is constant, and (c) the force constants do not change in the excited state, then the overlap for one specific normal mode $(k \mathrm{th})$ has the simple form for the harmonic poential surfaces

$$
\left\langle\phi_{k} \mid \phi_{k}(t)\right\rangle=\exp \left[-\frac{\Delta_{k}{ }^{2}}{2}\left(1-\exp \left(-i \omega_{k} t\right)\right)-\frac{i \omega_{k} t}{2}\right]
$$

where $\omega_{k}$ and $\Delta_{k}$ are respectively the vibrational frequency in $\mathrm{cm}^{-1}$ and the displacement of the $k$ th normal mode. In the case of many displaced normal modes, the total overlap is

$$
\langle\phi \mid \phi(t)\rangle=\prod_{k}\left\langle\phi_{k} \mid \phi_{k}(t)\right\rangle \exp \left(\frac{-i E_{0} t}{\hbar}-\Gamma^{2} t^{2}\right)
$$

where $E_{0}$ is the energy of the electronic origin and $\Gamma$ is a damping factor that determines the line width in the calculated spectrum. Thus, the complete overlap is

$$
\left.\exp _{\langle}^{\langle\phi \mid \phi(t)\rangle} \sum_{k}\left[-\frac{\Delta_{k}^{2}}{2}\left(1-\exp \left(-i \omega_{k} t\right)\right)-\frac{i \omega_{k} t}{2}\right]-\frac{i E_{0} t}{\hbar}-\Gamma^{2} t^{2}\right\}
$$

Calculation of the Fine Structure in the Emission and Absorption Spectra. The intensities and energies of the vibronic bands in the emission spectrum were calculated by using a set of values of $\Delta_{k}$, $\omega_{k}, \Gamma$, and $E_{0}$ and eqs $1-4 . E_{0}$ and $\Gamma$ were determined from the

(16) Tannor, D. J.; Heller, E. J. J. Chem. Phys. 1982, 77, 202.

(17) Larson, L. J.; Zink, J. I. Inorg. Chem. 1989, 28, 3519.

(18) Preston, D. M.; Shin, K. S.; Hollingsworth, G.; Zink, J. I. J. Mol. Siruct. 1988, 173, 185

(19) Zink, J, I. Coord. Chem. Rev. 1985, 64, 93.

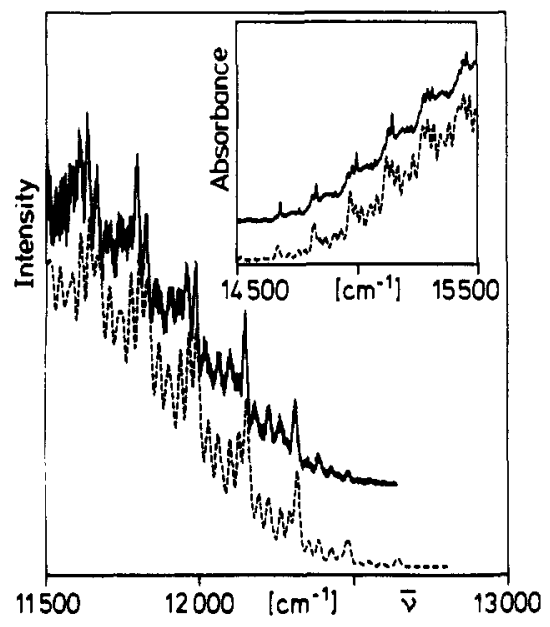

Figure 6. Comparison of the experimental (solid line) and calculated (dashed line) emission spectra of $\left[\left(\mathrm{C}_{2} \mathrm{H}_{5}\right)_{4} \mathrm{~N}\right]_{2}\left[\mathrm{Pd}(\mathrm{mnt})_{2}\right]$. The insert shows the experimental (solid line) and calculated (dashed line) lowenergy absorption band. The calculated absorption spectrum was obtained by using the same distortions as for emission.

Table II. Ground-State Vibrational Energies and Calculated Dimensionless Excited-State Distortions

\begin{tabular}{cccc}
\hline$h \omega, \mathrm{cm}^{-1}$ & $\Delta_{k}^{a}$ & $h \omega, \mathrm{cm}^{-1}$ & $\Delta_{k}{ }^{a}$ \\
\hline 52 & 0.90 & $210(207)^{b}$ & 0.35 \\
$93(85)^{b}$ & 1.10 & $222(222)^{c}$ & 0.37 \\
$126(103)^{b}$ & 1.00 & $236(233)^{b}$ & 0.49 \\
$162(160)^{c}$ & 3.12 & $298(319)^{b}$ & 0.41 \\
$188(180)^{b}$ & 1.47 & $342(349)^{c}$ & 0.35 \\
$200(207)^{b}$ & 0.40 & & \\
&
\end{tabular}

experimental spectrum. In the calculations discussed below, the value of $E_{0}$ was chosen to be $12642 \mathrm{~cm}^{-1}$, one quantum of the $162-\mathrm{cm}^{-1}$ mode higher in energy than the first clearly discernible peak at $12480 \mathrm{~cm}^{-1}$. Careful inspection of the experimental spectrum reveals weak but nonzero intensity between 12700 and $12500 \mathrm{~cm}^{-1}$. The best fit to the line widths was obtained by using a value of $\Gamma=5.5 \mathrm{~cm}^{-1}$. The values of the $\omega_{k}$ 's were obtained from the IR and Raman spectra with the exception of the $52-\mathrm{cm}^{-1}$ mode, for which no vibrational spectroscopic counterpart is known. This value was obtained from the emission spectrum. The values of the $\Delta_{k}$ 's were varied until the best fit between the calculated and experimental spectra was obtained.

The calculated and experimental emission spectra are shown in Figure 6. The agreement between the two is excellent. The theoretical spectrum was calculated by using the parameters given in Table II. Because all of the intensity and energy information in the emission spectrum was used, the uncertainties in the values of the parameters are small.

The largest distortion ( $31 \%$ of the sum of all of the $\Delta_{k}$ 's) occurs along the $162-\mathrm{cm}^{-1}$ mode. This mode forms the dominant progression in the spectrum. All of the other distortions are smaller by an order of magnitude. The dimensionless distortions given in Table II cannot readily be converted to bond length and bond angle changes in units of angstroms and degrees, respectively, because no normal-coordinate analysis of the molecule is available. The $162-\mathrm{cm}^{-1}$ mode is not a simple metal-sulfur stretch. ${ }^{6}$ Thus the emitting ${ }^{3} \mathrm{~B}_{3 \mathrm{~g}}$ state is distorted along both the $\mathrm{Pd}-\mathrm{S}$ bond lengths and the S-Pd-S bond angles in the excited state. Because the emission spectrum does not show any evidence of high-energy intraligand modes, the distortions within the ligand itself are small and its structure is very similar to that in the ground state.

The low-energy absorption band (Figure 3 ) has fine structure which is very similar to that of the emission spectrum (Figure 4). It was therefore tempting to calculate the absorption spectrum by using the distortions obtained from the emission spectrum fit.

(20) Adams, D. M.; Cornell, J. B. J. Chem. Soc, A 1968, 1299. 


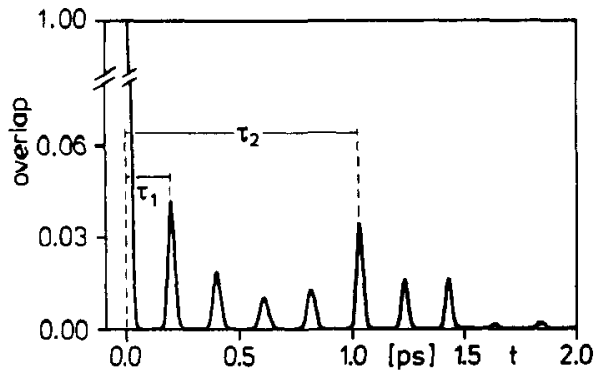

Figure 7. Time dependence of the magnitude of the overlap used in the calculation of the emission spectrum. Note the two distinct times $\tau_{1}(0.2$ ps) and $\tau_{2}(1 \mathrm{ps})$ which respectively correspond to the $162-\mathrm{cm}^{-1}$ separation between the most prominent bands and the $26-\mathrm{cm}^{-1}$ separation for the smaller sidebands.

All of the parameters in Table II were left unchanged with the exception of one vibrational frequency, that of the $162-\mathrm{cm}^{-1}$ mode, and $E_{0}$. The vibrational frequency in the excited electronic state was decreased to $150 \mathrm{~cm}^{-1}$, the value obtained from the absorption spectrum. $E_{0}$ was $14520 \mathrm{~cm}^{-1}$.

The experimental and calculated absorption spectra are compared in the insert to Figure 6 . The agreement in the low-energy region is good. These findings support the assignments of the two lowest electronic excited states made in the preceding section; both ${ }^{3} B_{3 g}$ (the emitting state) and ${ }^{1} B_{3 g}$ derive from the same orbital configuration and therefore are expected to have similar potential energy surfaces and show similar fine structure in the electronic spectra as is observed. The overall bandwidth is poorly reproduced, suggesting that more than one excited state is involved in the absorption band. Thus, the calculations of the vibronic structure provide insight into both the fine structure and the assignments of the lowest energy excited states.

Analysis of the Small Repetitive Spacings. The small energy differences between many of the vibronic features in the emission spectrum are exactly equal to the frequency differences between higher energy molecular vibrational modes. The time-dependent theoretical point of view provides a simple interpretation of the small spacings. ${ }^{21}$ The new insight arises from interpreting the

(21) Hollingsworth, G.; Shin, K. S.; Zink, J. I. Inorg. Chem. 1990, 29, 2501. system in the time domain. In the time domain, the overlap $\left\langle\phi_{k} \mid \phi_{k}(t)\right\rangle$ as a function of time for a given mode oscillates. The separation between the recurrences is a vibrational period. The total overlap is the product of the overlaps of each of the individual normal modes. Because each contributing mode has a different vibrational period, the product will be modulated and contain "beats". An analogue is found in sound waves. When the difference in frequencies between two sound waves is small compared to the sum of the two, the oscillation is rapid, but its "envelope" undergoes slow periodic variations, known as "beats". For two waves, the beat frequency is the difference between the frequencies of the waves.

The modulation of the overlap in the time domain can be clearly seen in Figure 7. This plot shows the overlap that gives the spectrum in Figure 6. The modulation that gives rise to the $26-\mathrm{cm}^{-1}$ separation will be emphasized in this discussion. At $t$ $=0$ the total overlap is 1 . The plot of the overlap versus time shows the initial falloff and subsequent recurrence at time $\tau_{l}=$ $0.2 \mathrm{ps}$. The $162-\mathrm{cm}^{-1}$ spacing in the frequency domain is equal to $2 \pi / \tau_{1}$. Most importantly, the magnitude of the overlap on successive recurrences rises and falls; i.e., the overlap is modulated. In Figure 7, the first maximum of the modulation occurs at the fifth recurrence, i.e., at $t=1 \mathrm{ps}$, labeled $\tau_{2}$. The $26-\mathrm{cm}^{-1}$ spacing in the emission spectrum of Figure 4 corresponds to $2 \pi / \tau_{2}$ in the time domain and is the difference in the frequencies of the 162 and $188-\mathrm{cm}^{-1}$ modes. In this case, the separation between the bands within a cluster is the difference in wavenumbers between the two most highly distorted modes in the excited electronic state.

The time domain picture used above provides a clear explanation of the features in the luminescence spectrum. Time-dependent theory is equivalent to the traditional Franck-Condon picture, which can also be used to interpret the vibronic spacings. For example, the peak at $12320 \mathrm{~cm}^{-1}$ (Figure 6) arises from two quanta of the $162-\mathrm{cm}^{-1}$ mode, and the lower energy sidebands on this peak arise from the combinations of two quanta of the 162 $\mathrm{cm}^{-1}$ mode and one quantum of the $52-\mathrm{cm}^{-1}$ mode, the $93-\mathrm{cm}^{-1}$ mode, or the $126-\mathrm{cm}^{-1}$ mode, respectively.

Acknowledgment. G.G. and W.G. thank the Fonds der Chemischen Industrie and J.I.Z. and C.R. thank the National Science Foundation (Grant CHE88-06775) for support of this work.

\section{Notes}

Contribution from the Department of Inorganic Chemistry, Chalmers University of Technology, S-41296 Göteborg, Sweden

\section{Preparation and Structural Characterization of $\mathrm{Cu}(\mathrm{CO}) \mathrm{Cl}$}

Mikael Hảkansson and Susan Jagner*

Received April 11, 1990

That solutions of copper(I) chloride in hydrochloric acid absorb carbon monoxide, yielding a white solid that readily loses $\mathrm{CO}$, has been known for well for over a century. ${ }^{1,2}$ The process has been recognized as the most satisfactory for reversible fixation of carbon monoxide under mild conditions, aromatic solvents containing, e.g., [ $\left[\mathrm{CuAlCl}_{4}\right]$ as the active component being employed industrially. 2,3 Various stoichiometries have been proposed for the solid precipitated from solutions of $\mathrm{CuCl}$ in hydrochloric

(1) Bruce, M. I. J. Organomet. Chem. 1972, 44, 209 and references therein.

(2) Pasquali, M.: Floriani, C. In Copper(I) Coordination Chemistry: Biochemical and Inorganic Perspectives; Karlin, K. D., Zubieta, J., Eds.; Adenine Press: New York, 1983; pp 311-330.

(3) Haase, D. J.: Walker, D. G. Chem. Eng. Prog. 1974, 70, 74. Walker. D. G. CHEMTECH 1975, 308. acid, i.e. $\mathrm{Cu}(\mathrm{CO}) \mathrm{Cl}, \mathrm{Cu}(\mathrm{CO}) \mathrm{Cl} \cdot 2 \mathrm{H}_{2} \mathrm{O}$, and $2 \mathrm{CuCl} \cdot \mathrm{CO} \cdot 2 \mathrm{H}_{2} \mathrm{O}$, investigation of the $\mathrm{CuCl} / \mathrm{CO}$ molar ratio in the precipitate by manometric methods indicating it to be anhydrous with the formula $\mathrm{Cu}(\mathrm{CO}) \mathrm{Cl}^{4}$ The solid-state structure of the compound formed when carbon monoxide is absorbed by solutions of chlorocuprates $(\mathrm{I})$ has been reported to be a halogen-bridged $(\mathrm{Cu}$ $\mathrm{ClCO})_{2}$ dimer, ${ }^{5}$ whereas the gold(I) analogue has been shown to be a linear monomer. 6 Infrared spectroscopic investigation of

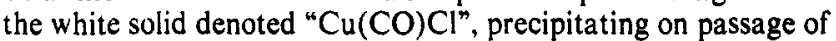
carbon monoxide through suspensions or solutions of copper(I) chloride in several different organic solvents, have shown that, whereas the $\mathrm{CO}$ band in solution varies slightly from solvent to solvent lying in the range $2070-2085 \mathrm{~cm}^{-1}$, the solid exhibits a band at $2120 \mathrm{~cm}^{-1}$ despite its origin. ${ }^{7}$ The higher frequency for the $\mathrm{CO}$ stretching vibration in the solid was interpreted as suggesting that copper(I) may have a coordination number lower than $4{ }^{7}$

(4) Backën, W.; Vestin, R. Acta Chem. Scand. 1979, A33, 85.

(5) Cotton, F. A.; Wilkinson, G. Advanced Inorganic Chemistry, 3rd ed.; Interscience: New York, 1972; p 911

(6) Jones, P. G. Z. Naturforsch. 1982, 37B, 823

(7) Pasquali, M.; Floriani, C.; Gaetani-Manfredotti, A. Inorg. Chem. 1981, 20,3382 . 